\title{
Zeta Potential
}

National Cancer Institute

\section{Source}

National Cancer Institute. Zeta Potential. NCI Thesaurus. Code C62354.

The magnitude of the electrical potential as generated by ion accumulation at the particulate surface. This surface consists of two layers, the Stern layer and the diffuse layer, which comprise an electrical double-layer. The measurement of the electrical potential provides important information regarding the dispersion mechanism, the degree of particle dispersion and the electrostatic potential of the sample. 